\title{
Ю.В. Крылов
}

\section{Проектирование волноводного трансформатора для широкополосного облучателя зеркальных антенн}

\begin{abstract}
Рассмотрены методы расчета волноводного трансформатора, показаны основные принципы проектирования данного устройства, входящего в состав ортомодового селектора частотно-поляризационного устройства облучателя зеркальных антенн или глобальных рупорных антенн. Проведен сравнительный анализ расчета мод, распространяющихся в трансформаторе, аналитическим методом и путем моделирования в CST Microwave Studio. При реализации ортомодового селектора по схеме с прямым высокочастотным выходом в трансформаторе возникают высшие типы волн, которые оказывают влияние на распространение волны основного типа. Из-за отсутствия решений данной проблемы при большом разносе частотных диапазонов приема и передачи представлен разработанный способ подавления влияния высших мод, возникающих в волноводе большего диаметра.

Ключевые слова: облучатель, ортомодовый селектор, волноводный трансформатор, высшие моды. doi: $10.21293 / 1818-0442-2016-19-3-16-20$
\end{abstract}

С развитием антенной техники спутниковой связи к облучателям зеркальных антенн или к глобальным рупорным антеннам предъявляются все более жесткие требования по ширине рабочих диапазонов частот, по работе с сигналами различной поляризации, а также совмещению каналов приема и передачи в одном облучателе. Все эти функции в облучателе выполняет входящий в его состав частотно-поляризационный селектор.

Существует несколько схем частотно-поляризационных селекторов [1], предназначенных для разделения сигналов приема и передачи с различными поляризациями, но все они имеют в своем составе ортомодовый селектор, представляющий собой волновод круглого сечения с поперечным крестовым разветвлением, служащим для разделения двух ортогональных плоскостей поляризации моды $H_{11}$. Из ортомодовых селекторов, в свою очередь, также можно выделить два типа. Они различаются лишь тем, какой сигнал рабочего диапазона частот (высокочастотный или низкочастотный) будет выделяться с торцевого (прямого) выхода ортомодового селектора. В зависимости от того, какой сигнал выделяется на прямом выходе селектора, значительно меняется форма волновода круглого сечения.

\section{Виды ортомодовых селекторов}

В ортомодовом селекторе, в котором обеспечивается выделение высокочастотного сигнала с прямого выхода селектора, необходимо спроектировать плавный переход между волноводом большего диаметра сечения и волноводом меньшего диаметра, тем самым сделав возможным прием сигнала на основном типе колебаний и отражение передающего сигнала в область крестового разветвления $[2,3]$.

По второму принципу построения ортомодового селектора при передаче низкочастотного сигнала к торцевому выходу селектора необходимо подключить фильтр нижних частот для отражения высокочастотного сигнала в область крестового разветвления. Ортомодовый селектор, построенный по такому принципу, обладает недостатком, а именно - узкополосностью фильтра нижних частот, по сравнению с селектором, у которого с прямого выхода выделяется высокочастотный сигнал. Таким образом, ввиду жестких требований, предъявляемых к современным спутникам связи, а именно по ширине рабочей полосы частот, данный способ построения ортомодового селектора представляется наименее перспективным.

При проектировании ортомодового селектора, с прямого выхода которого выделяется высокочастотный сигнал, необходимо иметь в виду, что при таком построении устройства в области волновода большего диаметра возникают высшие моды, которые, в свою очередь, оказывают негативное влияние при работе на основном типе волн $H_{11}$. В данной статье представлено исследование по подавлению влияния высших мод в ортомодовом селекторе с торцевым высокочастотным выходом.

Моделирование и расчет волноводного трансформатора

Внешний вид рассматриваемого ортомодового селектора показан на рис. 1. Данное устройство включает в себя: 1 - волновод большего диаметра; 2 - волновод меньшего диаметра; 3 - трансформатор круглого волновода; 4 - прямоугольный волновод.

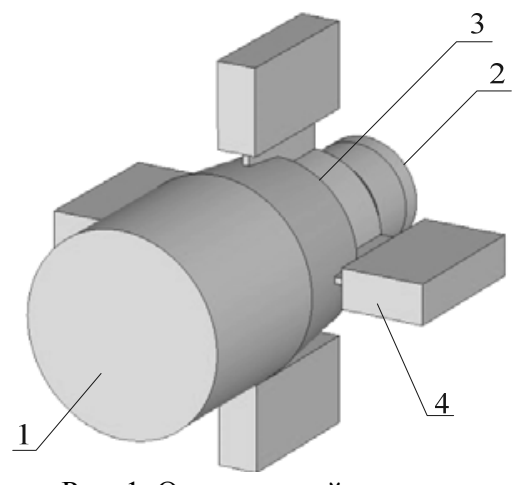

Рис. 1. Ортомодовый селектор

При использовании ортомодового селектора в облучателе зеркальной антенны к выходной части волновода 1 стыкуется рупор, другая сторона волновода соединяется с трансформатором 3, который 
служит для согласования волноводов с различными диаметрами сечений. Волноводный трансформатор 3 также служит для того, чтобы потери энергии основной волны $H_{11}$, возбуждаемой в волноводе 2 , на преобразование в волны высшего типа были минимальными. Крестовое разветвление, состоящее из четырех волноводов 4, предназначено для того, чтобы снять поляризационное вырождение волны $H_{11}$ в низкочастотном диапазоне частот. Проектирование такого селектора необходимо в первую очередь начинать с волноводного трансформатора 3, лишь затем проводить расчет согласующего трансформатора волновода прямоугольного сечения 4. Продольный вид трансформатора без ортогональных плеч из волноводов прямоугольного сечения показан на рис. 2 .

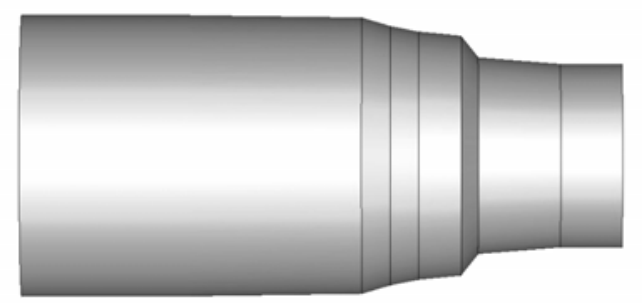

Рис. 2. Трансформатор волновода круглого сечения

Исследование волноводного трансформатора, показанного на рис. 2, проводилось в диапазонах частот: передача - 3,8-4,3 ГГц, прием - 5,9-6,4 ГГц. Моделирование осуществлялось в среде CST Microwave Studio (далее CST). Внутренний диаметр волновода большего диаметра, равный $d_{1}=50$ мм, был выбран исходя из условия

$$
\left(\lambda_{\text {кр }}\right)_{E_{01}} \leq \lambda_{0} \leq\left(\lambda_{\text {кр }}\right)_{H_{11}},
$$

где $\lambda_{0}=0,07$ м - длина волны, вычисленная на средней частоте диапазона 3,8-4,3 ГГц. Критические длины основной волны $H_{11}$ и ближайшей высшей волны $\mathrm{E}_{01}$ могут быть определены как

$$
\lambda_{\text {кр }}=\frac{\pi d}{q},
$$

где $d$ - диаметр волновода, $q=v_{m n}-$ корень производной функции Бесселя для волн $H_{m n}$, для волны $E_{m n} q=\varepsilon_{m n}-$ корень функции Бесселя [4].

Для волны $H_{11}\left(\lambda_{\text {кр }}\right)_{H_{11}}=1,71 \cdot d$, для волны $E_{01}$ $\left(\lambda_{\text {кр }}\right)_{E_{01}}=1,31 \cdot d$. Аналогично вычисляется диаметр меньшего волновода, который оказался равным $d_{2}=33$ мм . Длина переходного участка $L$ между волноводом большего диаметра и волноводом меньшего диаметра выбирается исходя из условия

$$
\lambda_{0}<L<2 \lambda_{0},
$$

где $\lambda_{0}=0,05 \mathrm{~m}$ - длина волны, вычисленная на средней частоте диапазона 5,9-6,4 ГГц.

Следующим шагом при проектировании волноводного трансформатора стал расчет типов и количества волн, распространяющихся на выходе трансформатора (со стороны большего диаметра волновода). В волноводе большего диаметра на частотах приема, кроме основной волны, неизбежно возбуж- даются и высшие типы волн, при расчете которых можно воспользоваться одним из параметров распространения той или иной моды. Первый из них постоянная распространения волн.

Постоянную распространения $\beta$ для волн $H_{m n}$ и $E_{m n}$ необходимо рассчитывать на самой высокой частоте рабочего диапазона $f_{0}=6,4$ ГГц . Для волн $H_{m n}$ она рассчитывается как

$$
\beta=\sqrt{k^{2}-\left(\frac{v_{m n}}{a}\right)^{2}},
$$

где $a$ - радиус волновода большего диаметра; $v_{m n}-$ корень производной функции Бесселя; $k$ - волновое число,

$$
k=\frac{2 \pi}{\lambda}=\frac{2 \pi f}{c} .
$$

Для волн $E_{m n}$ эта постоянная определяется по формуле

$$
\beta=\sqrt{k^{2}-\left(\frac{\varepsilon_{m n}}{a}\right)^{2}},
$$

где $\varepsilon_{m n}-$ корень функции Бесселя.

Из приведенных выражений для волн $H_{m n}$ и $E_{m n}$ несложно сделать вывод об условии распространения той или иной волны в волноводе. Для волн $H_{m n}$ условием распространения будет

$$
\frac{2 \pi f}{c}>\frac{v_{m n}}{a},
$$

для волн $E_{m n}-$ соответственно

$$
\frac{2 \pi f}{c}>\frac{\varepsilon_{m n}}{a} .
$$

Исходя из этих условий, можно определить, что в волноводе диаметром $d_{1}$ в высокочастотном рабочем диапазоне распространяются кроме основного типа волны $H_{11}$ еще высшие типы волн $E_{01}$ и $H_{21}$. Рассчитаем постоянные распространения для этих типов волн. Постоянная распространения для волны $H_{11}$ равна

$$
\beta=\sqrt{\left(\frac{2 \pi \cdot 6,4 \cdot 10^{9}}{3 \cdot 10^{8}}\right)^{2}-\left(\frac{1,841}{0,025}\right)^{2}}=1121 / \mathrm{m} .
$$

Постоянная распространения для волны $E_{01}$

$$
\beta=\sqrt{\left(\frac{2 \pi \cdot 6,4 \cdot 10^{9}}{3 \cdot 10^{8}}\right)^{2}-\left(\frac{2,405}{0,025}\right)^{2}}=93,34 \frac{1}{\mathrm{M}} .
$$

Постоянная распространения для волны $H_{21}$

$$
\beta=\sqrt{\left(\frac{2 \pi \cdot 6,4 \cdot 10^{9}}{3 \cdot 10^{8}}\right)^{2}-\left(\frac{3,054}{0,025}\right)^{2}}=55,171 / \mathrm{M} .
$$

При моделировании в CST трансформатора, показанного на рис. 2, в настройках выходного порта (порт, установленный на большем сечении диаметра волновода $d_{1}$ ) задавалось количество рассчитываемых мод, равное 10. Результаты расчета этих мод на частоте $f_{0}=6,4$ ГГц приведены на рис. 3 . 


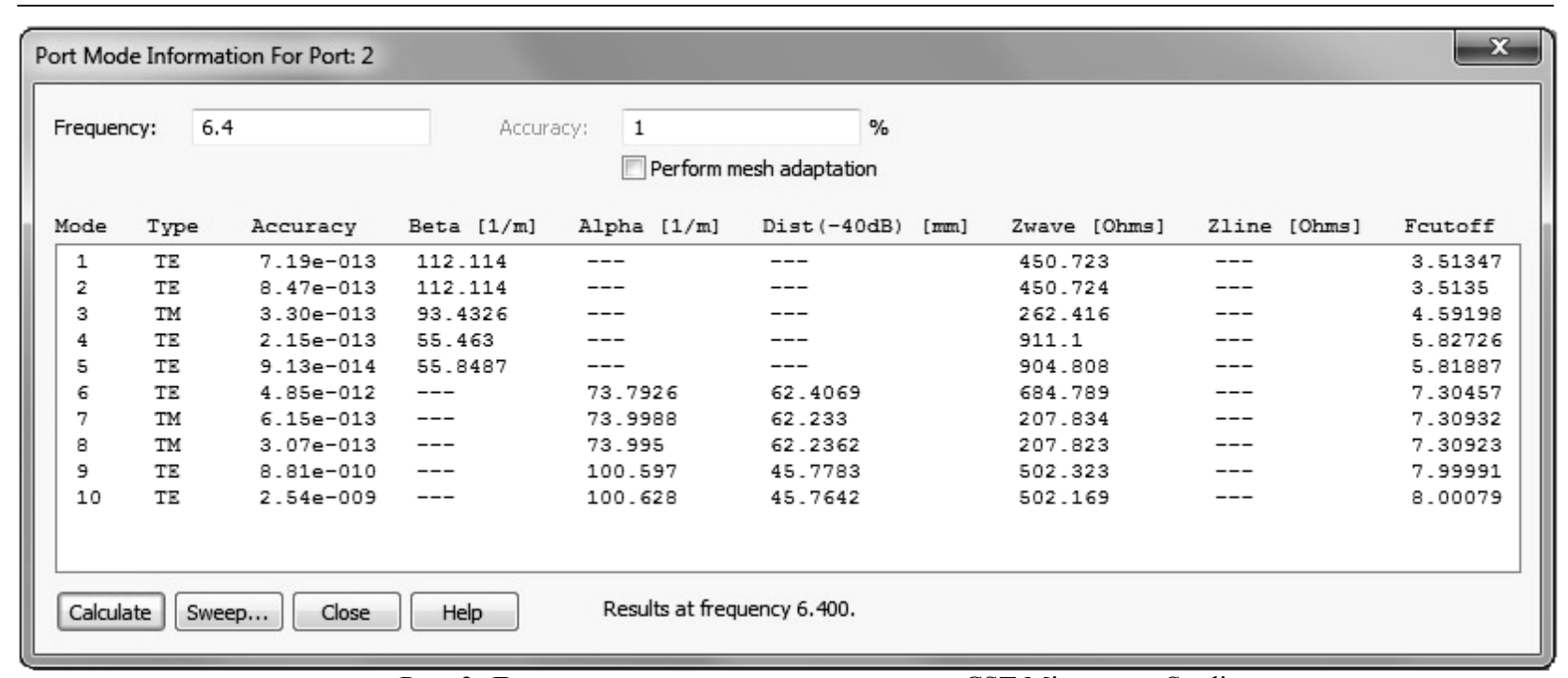

Рис. 3. Диалоговое окно расчета мод в среде CST Microwave Studio

Для удобства сравнения результаты моделирования и аналитических расчетов сведены в таблице. Из таблицы видно, что 1-я и 2-я мода, рассчитанные в CST, соответствует волне $H_{11}, 3$-я мода - волне $E_{01}$, 4-я и 5-я мода - волне $E_{01}$. Расхождение результатов расчетов постоянных распространения $\beta$ аналитическим методом и моделированием - минимально. Из таблицы можно заметить, что в CST значения постоянных распространения $\beta$ как 1-й и 2-й мод, так и 4-й и 5-й, совпадают, и это связано с вырождением волн $H_{11}$ и $H_{21}$ соответственно. Волны $H_{11}$ и $H_{21}$ имеют две плоскости поляризации, поэтому они могут быть ориентированы на выходе устройства двумя способами. Это также можно заметить, сравнив диаграммы поля 1-й и 2-й мод, 4-й и 5-й мод в поперечном сечении волновода, показанного на рис. 4.

Сравнение результатов расчета и моделирования

\begin{tabular}{|c|c|c|c|c|}
\hline \multirow{2}{*}{ Мода } & \multicolumn{2}{|c|}{ Моделирование } & \multicolumn{2}{c|}{ Расчет } \\
\cline { 2 - 5 } & Beta, $1 / \mathrm{m}$ & $F_{\text {cutoff, }}$ ГГц & $\beta, 1 / \mathrm{M}$ & $f_{\text {кр }}$, ГГц \\
\hline 1 & 112,114 & 3,51347 & 112,0 & 3,516 \\
\hline 2 & 112,114 & 3,5135 & 112,0 & 3,516 \\
\hline 3 & 93,4326 & 4,59198 & 93,34 & 4,595 \\
\hline 4 & 55,463 & 5,82726 & 55,17 & 5,832 \\
\hline 5 & 55,8487 & 5,81887 & 55,17 & 5,832 \\
\hline 6 & ----- & 7,30457 & ----- & 7,318 \\
\hline
\end{tabular}

Другим критерием при расчете типов волн, распространяющихся в волноводе, могут быть критические частоты волн. Распространяться будут только те моды, критические частоты которых будут меньше рабочей частоты, в данном случае ниже самой верхней частоты $f_{0}=6,4$ ГГц рабочего диапазона. Для волн $E_{m n}$ критическая частота определяется как

$$
f_{\text {кр }}=\frac{\varepsilon_{m n} c}{2 \pi a},
$$

для волн $H_{m n}$

$$
f_{\text {кр }}=\frac{v_{m n} c}{2 \pi a} .
$$

Рассчитаем критические частоты для волны $H_{11}$, для высших типов волн $E_{01}$ и $H_{21}$, а также для волны $H_{01}$ :

$$
\begin{gathered}
f_{\text {кр } H_{11}}=\frac{1,841 \cdot 3 \cdot 10^{8}}{2 \pi \cdot 0,025}=3,516 \text { ГГц . } \\
f_{\text {кр } E_{01}}=\frac{2,405 \cdot 3 \cdot 10^{8}}{2 \pi \cdot 0,025}=4,595 \text { ГГц . } \\
f_{\text {кр } H_{21}}=\frac{3,054 \cdot 3 \cdot 10^{8}}{2 \pi \cdot 0,025}=5,832 \text { ГГц . } \\
f_{\text {кр } H_{01}}=\frac{3,832 \cdot 3 \cdot 10^{8}}{2 \pi \cdot 0,025}=7,318 \text { ГГц . }
\end{gathered}
$$

Как можно заметить по результатам расчета критических частот, в волноводе будут распространяться только волны $H_{11}, E_{01}, H_{21}$, ближайшая волна высшего типа $H_{01}$ распространяться не будет, т.к. $f_{\text {кр } H_{01}}>f_{0}$. Это также можно проследить по рис. 4, из которого видно, что шестая мода не распространяется по волноводу, а затухает сразу на входе порта. Результаты расчета критических частот также совпадают с результатами моделирования (см. рис. 3 и таблицу). Таким образом, при расчете количества распространяющихся мод в проектируемом трансформаторе можно ограничиться одним из вышерассматриваемых условий, связанных с критическими частотами волн или с их постоянными распространения.

\section{Подавление влияния высших мод}

При проектировании трансформатора, для лучшего согласования двух волноводов с диаметрами $d_{1}$ и $d_{2}$ было решено разбить переходную секцию на пять ступеней разной длины и разными диаметрами сечений. Критерием должного согласования трансформатора будет служить величина обратных потерь уровнем менее минус 20 дБ в полосе частот 5,9-6,4 ГГц. Так как данное устройство входит в состав частотно-поляризационного селектора облучателя зеркальных антенн или глобальных рупорных антенн, то в большинстве случаев необходимо, что- 
бы работа селектора осуществлялась на основной волне $H_{11}$ во всем рабочем диапазоне частот. В расчетах, приведенных выше, было показано, что кроме основного типа волны $H_{11}$ на выходе волновода большего сечения распространяются еще высшие типы волн $E_{01}$ и $H_{21}$. Для стабильной работы как в самом устройстве, так и в облучателе в целом необходимо, чтобы волна $H_{11}$ распространялась с наименьшими потерями. Чтобы выполнить данное требование, необходимо, чтобы волноводный трансформатор обеспечивал не только согласование волноводов разного сечения на основной моде, но и подавлял влияние высших мод. Для того чтобы оценить влияние этих мод, необходимо при моделировании следить за развязкой между 1-й модой на входе трансформатора (со стороны меньшего сечения) и 3-5-й модами на выходе трансформатора. Для подавления вышеуказанных мод необходимо варьировать диаметрами сечений ступеней переходной секции, а также их длинами.
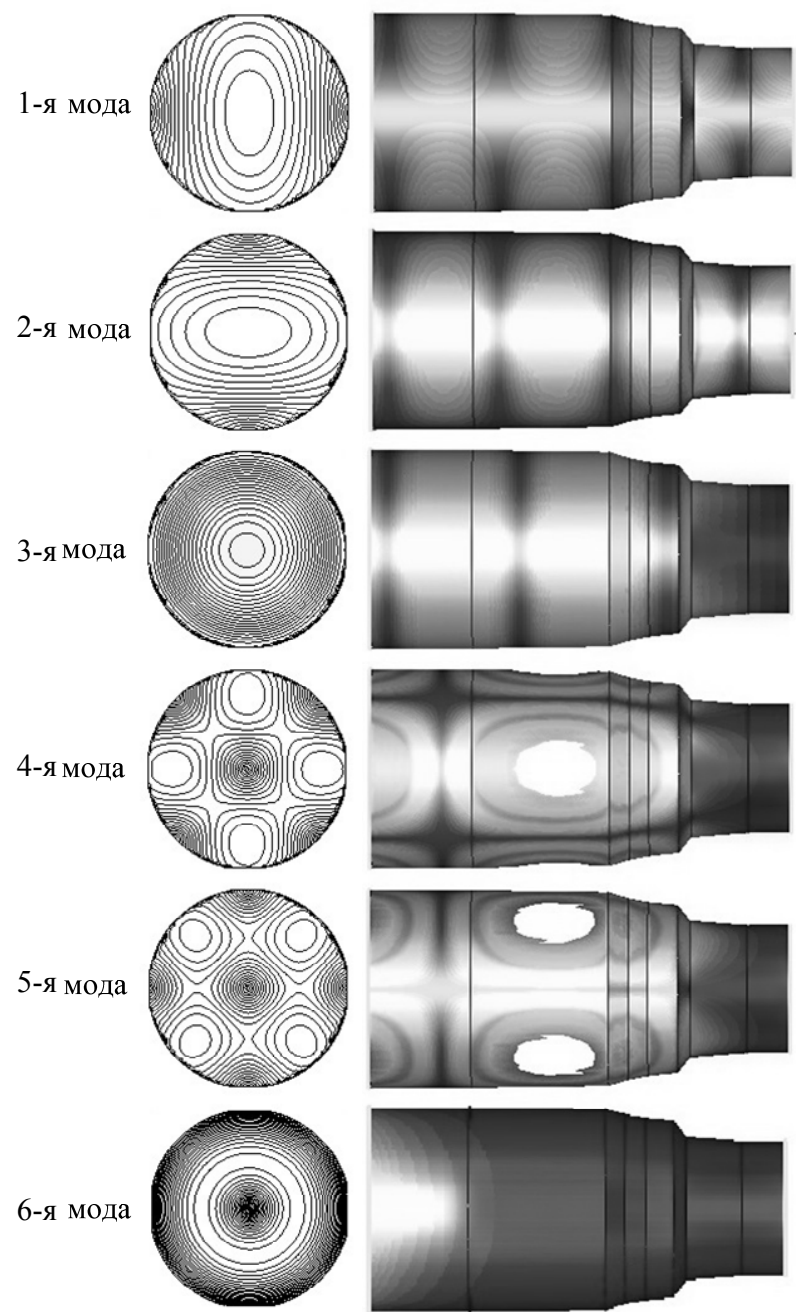

Рис. 4. Диаграммы полей в волноводном трансформаторе

Критериями настройки волноводного трансформатора будут служить величина обратных потерь менее минус 20 дБ в полосе частот 5,9-6,4 ГГц на входе устройства и величина развязки между основной модой и высшими менее минус 30 дБ. Спроек- тированный волноводный трансформатор, удовлетворяющий этим требованиям, показан на рис. 2. Как уже говорилось, устройство состоит из пяти ступеней, поверхности которых представляют собой усеченные конусы с разными диаметрами сечений. За счет такой геометрии устройства обеспечивается подавление влияния высших мод. За этим можно проследить, посмотрев на рис. 4 диаграммы поля для различных мод в поперечном и продольном разрезе волновода.

Из рис. 4 видно, что в волноводе распространяются только две первые моды, которые соответствуют вырожденной волне $H_{11}$ с ортогональными плоскостями поляризации, остальные моды подавляются в узкой части трансформатора за счет того, что волновод с меньшим диаметром является запредельным для волн $E_{01}, H_{21}$. Оценить же влияние высших мод, оказываемое на распространение основной волны $H_{11}$, можно с помощью рис. 5 и 6, на которых показаны результаты моделирования трансформатора.

\section{Результаты моделирования}

На рис. 5 показаны графики прямых и обратных потерь в полосе частот 5,9-6,4 ГГц. Обратные потери в рабочем диапазоне частот составили менее минус 22 дБ, прямые потери - более минус 0,03 дБ, что соответствует требованиям, предъявляемым к данному устройству.

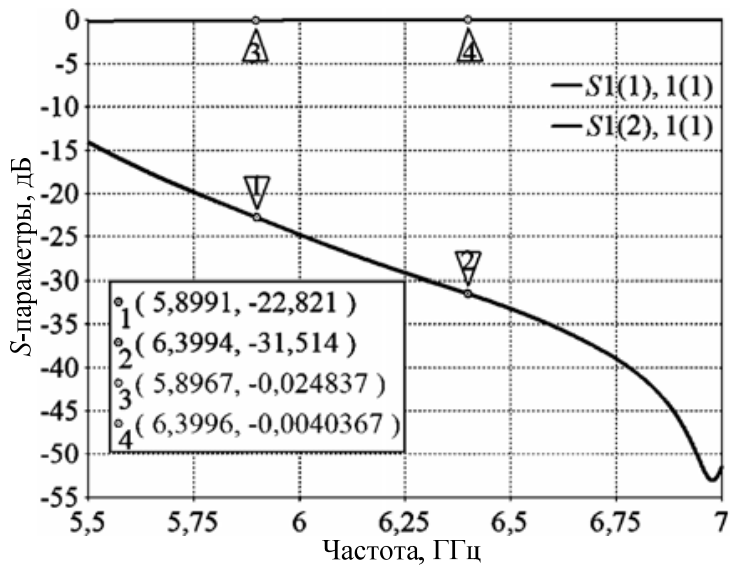

Рис. 5. Частотные зависимости потерь на отражение $S_{11}$ и на прохождение $S_{21}$ в волноводном трансформаторе

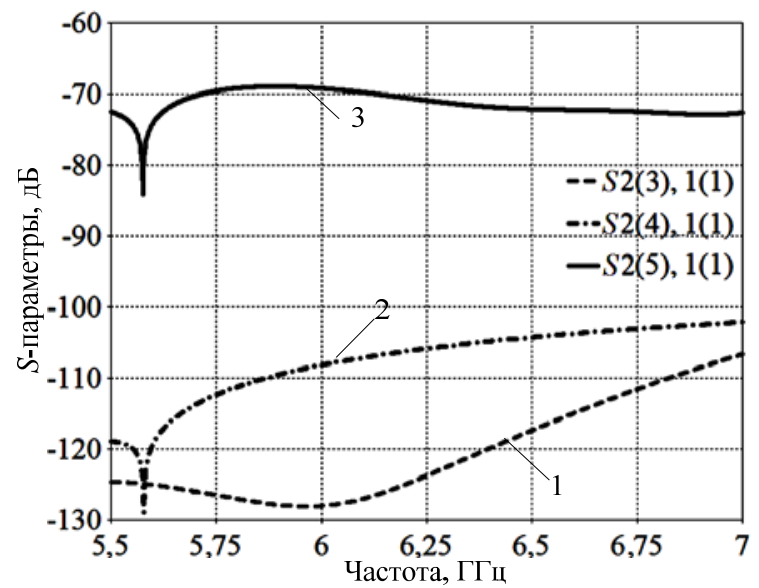

Рис. 6. Развязки между первой и высшими модами 
На рис. 6 показаны графики развязок, где 1 развязка между 1-й модой на входе трансформатора (со стороны меньшего сечения) и 3-й модой на выходе трансформатора; 2 - развязка между 1-й и 4-й модами; 3 - развязка между 1-й и 5-й модами. Из рис. 6 видно, что подавление всех мод составляет менее -68 дБ, что более чем достаточно для того, чтобы нивелировать влияние высших мод на распространение основной волны $H_{11}$ в диапазоне частот приема.

\section{Заключение}

В статье были рассмотрены методы расчета волноводного трансформатора, показана последовательность действий при проектировании данного устройства, входящего в состав частотно-поляризационного селектора облучателя зеркальных антенн или глобальных рупорных антенн. Проведен сравнительный анализ расчета мод, распространяющихся в трансформаторе, аналитическим методом и путем моделирования в CST Microwave Studio. Представлен разработанный способ подавления влияния высших мод, возникающих при переходе на волновод большего диаметра. Подавление осуществляется волноводным трансформатором, состоящим из нескольких ступеней в виде усеченных конусов. Работоспособность такого способа проектирования волноводного трансформатора подтверждается результатами моделирования, а именно развязками между 1-й модой на входе трансформатора (со стороны меньшего сечения) и высшими модами на выходе трансформатора, которые составляют менее минус 68 дБ. Этого достаточно для того, чтобы минимизировать влияние высших мод на распространение основного типа волны.

\section{Литература}

1. Крылов Ю.В. Частотно-поляризационная селекция сигналов в рупорных облучающих системах зеркальных антенн // Исследования наукограда. - 2015. - № 2. - С. 5-9.
2. Компактный облучатель Ка/Q-диапазона круговой поляризации / Ю.В. Крылов, И.Ю. Данилов, Ю.Г. Выгонский, А.Г. Романов // Наукоемкие технологии. - 2015. Вып. 3, № 16. - С. 52-55.

3. Крылов Ю.В. Проектирование облучателя в Ка/Qдиапазоне на основе «восстанавливающей» схемы / Ю.В. Крылов, В.Б. Тайгин // Вестник СибГАУ. - 2015. Вып. 2, №16. - С. 417-422.

4. Фельдштейн А.Л. Справочник по элементам волноводной техники / А.Л. Фельдштейн, Л.Р. Явич, В.П. Смирнов. - М.: Связь, 1967. - С. 140-149.

\section{Крылов Юрий Валерьевич}

Инженер-конструктор,

АО «Информационные спутниковые системы» им. акад. М.Ф. Решетнева», г. Железногорск

Тел.: +7 (391-9) 72-24-39

Эл. почта: unker007@mail.ru

Krylov Y.V.

Design of waveguide transformer for broadband feed of reflector antennas

The methods of calculating the waveguide transformer, showing the basic principles of the design of the device, which is part of the frequency-polarization selector of the feed reflector antennas or global horn antennas are presented. A comparative analysis of the calculation of modes distributed in the transformer by the analytical method and simulation in CST Microwave Studio is carried out. The developed method of suppressing the influence of higher order modes, resulting in a larger diameter of waveguide is described.

Keywords: feed of reflector antennas, orthomode transducer, waveguide transformer, higher modes. 